from the weight of the surgical sponge used, and postoperative bleeding was measured via the comparison of preoperative and postoperative hematocrit and hemoglobin levels. The mean perioperative blood loss in the mTRP group was significantly lower than that in the transvesical prostatectomy group $(362.5 \mathrm{ml}$ versus $640.0 \mathrm{ml}, P=0.002)$. Hematocrit and hemoglobin levels decreased after surgery in both groups, but the decreases were smaller in the mTRP group $(P=0.004$ and $P=0.020$, respectively).

The authors acknowledge that it is difficult to measure perioperative bleeding accurately; however, all procedures were performed by only three surgeons, so it is likely that the observed differences in blood loss were related to the surgical techniques used.

Original article Dall'Oglio MF et al. (2006) An improved technique for controlling bleeding during simple retropubic prostatectomy: a randomized controlled study. BJU Int $\mathbf{9 8 :}$ 384-387

\section{Severity of erectile dysfunction is related to extent of coronary disease}

Researchers in Italy have established a link between erectile dysfunction and coronary atherosclerotic burden. Montorsi et al. allocated 285 patients with coronary artery disease (CAD) into three equal groups: Group 1, patients with acute coronary syndrome (ACS) and single-vessel disease; Group 2, patients with ACS and 2-3-vessel disease; Group 3, patients with chronic coronary syndrome (CCS). A control group comprised 95 patients with normal arteries.

The prevalence of erectile dysfunction was substantially lower in Group 1 than in Group 3 (22\% vs $65 \%$ ) and was similar to that in the control group. Atherosclerotic burden was also lower in Group 1 than in Group 3 (2 vs 40 as measured by Gensini's score). The prevalence of dysfunction and atherosclerotic burden in Group 2 were similar to those in Group 3, suggesting that, for patients with ACS, the prevalence of erectile dysfunction was related to atherosclerotic burden. In patients with CCS, however, the extent of atherosclerosis was not related to the prevalence of erectile dysfunction. The severity of erectile dysfunction correlated with atherosclerotic extent. In Group 3 patients, erectile dysfunction preceded the onset of CAD by a mean interval of 24 months. Age, multivessel disease and CCS were found to be independent predictors of erectile dysfunction. Conversely, for patients with ACS, erectile dysfunction was associated with a fourfold increase in risk of multivessel disease.

The authors state that erectile dysfunction could be a marker for CAD, as the condition frequently precedes onset of CAD.

Original article Montorsi $\mathrm{P}$ et al. (2006) Association between erectile dysfunction and coronary artery disease. Role of coronary clinical presentation and extent of coronary vessels involvement: the COBRA trial. Eur Heart J

[doi: 10.1093/eurheartj/ehl142]

\section{High-volume surgeons have lowest radical prostatectomy costs}

The relationship between patient volume and hospital costs has been assessed for many surgical procedures, and evidence suggests that high-volume surgeons have reduced total costs. Few data exist, however, on the effect of patient volume on hospital costs for radical prostatectomy operations. Ramirez et al. have analyzed this relationship for radical prostatectomies carried out in Florida, US.

In $1998,3,167$ radical prostatectomies were performed in Florida. Patient volumes per surgeon were in the range 2-162 operations (mean 68, SD 41), and hospital charges were US $\$ 4,755-\$ 140,201$ (mean $\$ 18,200$, median $\$ 16,600)$. There was an inverse correlation between patient volume and overall costs. Of the variables analyzed, volume of patients was the only important factor; age, ethnicity and comorbidity had little influence on costs. Multivariate analysis suggested that each additional radical prostatectomy resulted in a $\$ 25$ decrease in hospital costs; the difference in costs for a surgeon who performed 50 radical prostatectomies annually compared with one who performed 10 procedures would be $\$ 1,000$-corresponding to a saving of $\$ 1,000,000$ for a hypothetical group of 1,000 patients. In 1997, the average volume per surgeon for this operation was 18 procedures.

The findings suggest that radical prostatectomy operations should be redistributed from low-volume to high-volume surgeons. Logistic, geographic and motivational factors mean, 